\title{
Segurança ambiental na SADC: novas perspectivas \\ teóricas de análise
}

Ricardo Margalho Prins ${ }^{1}$

Resumo: Diante da vasta gama de novos temas na agenda internacional, as discussões sobre segurança assumem as mais variadas formas, cada vez mais se distanciando da tradicional visão westfaliana do sistema internacional, com novas possibilidades de ameaça. As discussões sobre segurança ambiental, embora recentes, têm figurado com maior frequência no meio acadêmico, sobretudo nos teóricos da chamada Escola de Copenhague. O objetivo deste trabalho é o de analisar as mudanças climáticas na região da Comunidade para o Desenvolvimento da África Austral (SADC) do ponto de vista da segurança ambiental, buscando responder à seguinte questão: há elementos que permitam considerar meio-ambiente como questão de segurança na região? A busca pela resposta positiva desta questão será feita por análise qualitativa de dados quantitativos, através de análise de conteúdo de relatórios e documentos sobre o SADC.

Palavras-chave: Meio-ambiente. Segurança ambiental. SADC.

Abstract: In face of the wide array of new subjects in the international agenda, discussions about security are increasingly diverse, each time going further from the traditional Westphalian view of the international system, with new threat possibilities. Discussions about environmental security, although recent, are more often present in academic texts, especially in those from the so-called Copenhagen School. The purpose of this article is to analyze the climate changes in the Southern African Development Community (SADC) from the standpoint of the environmental security, seeking to answer the following question: are there elements that allow considering environment as a security issue in that region? The answer to this question will use mixed methods -

Graduando em Relações Internacionais no Centro Universitário Internacional (UNINTER), Pesquisador do Núcleo de Pesquisa em Relações Internacionais NEPRI/UFPR. Endereço eletrônico: ricardoprins@ gmail.com. 
qualitative analysis of quantitative data - through contente analysis of both reports and documents about the SADC.

Keywords: Environment. Environmental security. SADC.

\section{Introdução}

O mundo mudou. Ainda que naturalmente óbvias afirmações como esta, ao serem consideradas no universo científico, requerem comprovações muito mais substanciais para terem validade. Assim como muda o mundo, mudam as concepções sobre o mundo. Sartre discorre com maestria sobre os variados 'saberes', visões de mundo ou filosofias: "Reduzido à sua mais simples expressão, o objeto filosófico permanecerá no 'espírito objetivo' sob forma de ideia reguladora indicando uma tarefa infinita; assim, fala-se hoje, entre nós, da 'ideia kantiana' ou, entre os alemães, da Weltanschauung de Fichte" (SARTRE, 1960). Tais mudanças na visão do mundo oferecem aos estudos de segurança interessantes mudanças de paradigma, na medida em que com o passar dos anos, outros assuntos são incorporados à agenda de discussões. Buzan e Hansen oferecem uma interessante perspectiva sobre a evolução dos estudos de segurança no primeiro capítulo de seu livro sobre o tema, ao reforçarem a ideia de que a compreensão de segurança muda com o passar dos anos. (BUZAN; HANSEN, 2008).

A inserção de novos temas na agenda dos estudos de segurança 
foi discutida amplamente a partir do fim dos anos 80 (BUZAN; HANSEN, 2008, p. 187-192), sobretudo pelos teóricos da chamada Escola de Copenhague, com sua teoria da securitização. Este trabalho buscará discutir as possibilidades de inserção de discussões de meioambiente na pauta da segurança, por meio da análise do caso da Southern African Development Community, ou Comunidade para o Desenvolvimento da África Austral (SADC). Ao serem examinados alguns documentos e estudos sobre a organização, pode-se buscar enriquecer as discussões na área de 'segurança ambiental' - como é chamada a confluência entre segurança e meio-ambiente (MATTHEW; MCDONALD, 2004).

O objetivo deste trabalho é o de analisar as mudanças climáticas na região da Comunidade para o Desenvolvimento da África Austral (SADC) do ponto de vista da segurança ambiental, buscando responder à seguinte questão: há elementos que permitam considerar meioambiente como questão de segurança na região? A busca pela resposta positiva desta questão será feita por análise qualitativa de dados quantitativos, através de análise de conteúdo de relatórios e documentos sobre o SADC. A primeira seção irá abordar questões teóricas referentes à questão do meio-ambiente e da segurança, sendo seguida pela segunda, que irá tratar da SADC. A terceira seção trará dados e informações sobre a SADC, relacionando-os com a questão da segurança ambiental. 


\section{Ordem internacional, meio-ambiente e segurança.}

Com as mudanças ocorridas no mundo e a subsequente inserção de novos temas na agenda de discussão do sistema internacional, faz-se necessária a elaboração de todo um cabedal teórico que ofereça aos analistas as ferramentas necessárias para oferecerem suas perspectivas a partir da observação das questões apresentadas pelo desenrolar dos fatos no mundo. Barros-Platiau, Varella e Schleicher tratam de três destas abordagens, no que tange à questão dos problemas do meio ambiente: abordagens organizacionais, regimes internacionais e governança global (BARROS-PLATIAU; VARELLA; SCHLEICHER, 2004).

Estes problemas do meio-ambiente são tratados pelos decision makers de variadas formas. Esta é outra questão observada pelos analistas, que irão buscar discutir se as ações tomadas pelos decision makers oferecem alguma eficácia (ver MEDEIROS; STEINER, 2010), além da eficácia das intervenções de atores não-estatais nas discussões sobre meio-ambiente (ver VILLA, 2001).

Sem um amplo aprofundamento acerca das possibilidades teóricas dentro da questão do meio-ambiente, buscar-se-á aqui apenas trazer à tona outra realidade que se observa com as mudanças no sistema internacional: a interação entre segurança internacional e meio- 
ambiente.

Com as mudanças no sistema internacional consolidadas no fim da década de 1990, que marcaram a transição de um modelo westfaliano de Estado para uma "Ordem Mundial” (FALK, 2004, p.28), mudam-se também conceitos e a percepção da nova realidade internacional. Surgem novas formas de organização política no sistema internacional e também um novo modelo que deixa de privilegiar a "globalização militar" para privilegiar a "globalização econômica" (FALK, 2004; MITTELMAN, 2010). Os efeitos desta mudança causam impacto tanto na organização política internacional quanto na vida social dos indivíduos. Mittelman ressalta um movimento de resistência ao neoliberalismo econômico por meio da sociedade civil, fazendo surgir o hiperconflito (MITTELMAN, 2010).

O modelo econômico global resultante do processo do hiperconflito e da hipercompetição (vale notar que este modelo é fundamentado no medo que, segundo Bauman é a "ignorância da ameaça e do que deve ser feito - do que pode e do que não pode - para fazê-la parar ou enfrentá-la" (BAUMAN, 2008, p.8) utiliza-se de preceitos hobbesianos para conduzir o comércio internacional. A constituição da soberania dentro do estado de natureza hobbesiana é feita por relações de poder fundadas no medo: "a soberania se forma sempre por baixo, pela vontade daqueles que tem medo" (FOUCAULT, 2010 , p.81). Este modelo fundamentado pelo medo faz com que a 
ordem internacional resultante seja fundada na insegurança, fazendo com que o imperativo da segurança nacional se afirme como técnica da arte de governar, na qual o Estado assume para si a função e o poder de fabricar a liberdade - e garantir a segurança (FOUCAULT, 2008).

Dentro deste contexto, nos resta observar o papel do meioambiente na estruturação destas novas ameaças. Com o crescente impacto da degradação ambiental e as consequências das mudanças climáticas, houve uma expansão na associação desses impactos com noções não-convencionais de segurança, o que fez com que o meioambiente passasse a ser considerado como fonte de ameaça à segurança individual, nacional ou global (BISWAS, 2011).

No entanto, esta identificação entre os temas demanda uma abordagem interdisciplinar: especialistas em meio-ambiente e em segurança, buscam entender e correlacionar fatores causais de ameaças ambientais à segurança, oferecendo novas perspectivas aos estudos de segurança (BISWAS, 2011).

\section{Comunidade para o Desenvolvimento da África Austral}

Dentre as variadas iniciativas de integração regional, este trabalho irá abordar a iniciativa da Southern African Development Community - Comunidade para o Desenvolvimento da África Austral (SADC). Esta seção irá tratar de algumas características gerais desta 
organização, visando facilitar a discussão posterior a respeito da relação entre a segurança e o meio-ambiente dentro do contexto anteriormente delimitado.

A SADC surge em 1992, com o compromisso e o objetivo de estabelecer uma comunidade de desenvolvimento na região. Seu tratado de fundação foi assinado por Angola, Botswana, Lesoto, Malawi, Moçambique, Namíbia, Suazilândia, Tanzânia, Zâmbia e Zimbabwe. O documento entende comunidade como "organização para integração econômica" (SADC, 1992, p.4), tendo oito objetivos principais, dentre os quais o primeiro é atingir "desenvolvimento e crescimento econômico, aliviar a pobreza, aumentar o padrão e a qualidade de vida das pessoas da África Austral e dar suporte aos deficientes sociais por meio da integração regional" (SADC, 1992, p.5); o terceiro é "promover e defender a paz e a segurança" (SADC, 1992, p.5); e o sétimo é "alcançar utilização sustentável de recursos naturais e proteção efetiva do meio-ambiente" (SADC, 1992, p.5). São ressaltados estes três dentre os sete em razão do tema do artigo, apenas para reforçar a existência das preocupações tanto com segurança quanto com meioambiente dentro da organização.

Seu tratado afirma que a consecução destes objetivos far-se-á por meio de políticas públicas inter e intra-estatais, tendo por obrigação o compromisso de cada país-membro em adotar medidas que não sejam contrárias à promoção dos objetivos do SADC (SADC, 1992, p.6). 
Como o trabalho visa analisar apenas aspectos conjunturais, dispensamse maiores detalhamentos sobre a estrutura institucional do organismo. Atualmente são membros: Angola, Botswana, República Democrática do Congo, Lesoto, Madagascar, Malawi, Ilhas Maurício, Moçambique, Namíbia, Seychelles, África do Sul, Suazilândia, Tanzânia, Zâmbia e Zimbabwe (SADC, 2012).

\section{Meio-ambiente e segurança na SADC}

O estabelecimento da relação entre segurança e meio-ambiente será feito com base em um estudo feito por Larry Swatuk, que classifica as possibilidades de mudança ambiental em três tipos: macro, meso e micro (SWATUK, 2007). Após a exposição das possibilidades, será feita uma relação entre as mudanças ambientais e a segurança, considerando-se ainda o Índice de Desenvolvimento Humano dos países da SADC como variável interveniente nessa relação.

Para efeitos de análise convém classificar, conforme sugere Swatuk (SWATUK, 2007, p. 26), as mudanças ambientais em macro, meso e micro. Reconhece-se os potenciais desdobramentos desta classificação, principalmente em relação aos diferentes graus de percepção das mudanças enquanto ameaça por cada Estado e dos diferentes tipos de impacto provenientes das mudanças, bem como quanto às diferentes capacidades de cada Estado, que dão a cada um 
maior ou menor grau de resiliência em relação às mudanças climáticas. Enquanto, por um lado, sugere-se o aprofundamento em relação às percepções e impactos em outro estudo, optou-se aqui por se utilizar do IDH como fator interveniente em relação às capacidades de resiliência de cada Estado.

TABELA 1 - Graus de mudança ambiental

\begin{tabular}{lll}
\hline \multicolumn{1}{c}{ Macro } & \multicolumn{1}{c}{ Meso } & \multicolumn{1}{c}{ Micro } \\
\hline Alteração de ciclos & Perda de florestas; & Pontos de poluição; \\
hidrológicos; & Perda de espécies; & Mudanças de correntes; \\
Enchentes e secas; & Redução no número de & Perda de colheitas; \\
Mudanças no Deserto do & peixes; & Decaimento urbano. \\
Kalahari. & Mudanças na costa; & \\
& Mudanças no solo; & \\
& Desertificação. & \\
\hline
\end{tabular}

Fonte: SWATUK, 2007, p. 26-29

De acordo com Swatuk, podem ser observados os seguintes impactos, de acordo com os graus de mudança ambiental: com relação ao grupo macro, observa-se um grande número de ocorrências de enchentes e secas em vários pontos da região da África Austral em face das recentes mudanças climáticas mundiais, porém com uma postura reativa por parte da SADC. Além disso, vários modelos trazem um complexo cenário para as mudanças na região do Kalahari (que se 
estende de uma parte da África do Sul até o sul do Camarões), com grandes impactos em potencial, principalmente em razão das atividades econômicas desempenhadas na região, que podem desencadear grandes "grupos de reassentamento de refugiados ambientais", que, por sua vez, são fontes em potencial de conflito (SWATUK, 2007, p. 30-31).

Para o grupo meso, já são observáveis impactos econômicos em face do esgotamento de recursos naturais. Assim como para as mudanças macro, o impacto das mudanças meso para a África Austral é uma questão vital (principalmente a perda de florestas e espécies e o processo de desertificação), causada principalmente por uma configuração artificialmente construída (intervenção humana no meioambiente) pela ação de grupos de grandes empresas. Os impactos observáveis (perda de biodiversidade, aumento de endividamento em função da incapacidade de se desempenhar atividades econômicas que outrora eram fundamentais para as regiões, migração populacional). Embora as mudanças meso sejam mais facilmente reversíveis, há uma tendência evasiva por parte dos decision makers em formular políticas visando amenizar as causas ambientais dos problemas econômicos da região, em face de grupos de interesse de opinião contrária (SWATUK, 2007, p.32).

As mudanças micro, de acordo com Swatuk, impactam principalmente populações mais pobres da região, embora ressaltando a grande adaptabilidade da região, que faz com que, por muitas vezes, os 
indivíduos impactados pelas mudanças consigam se adaptar e sobreviver a elas (SWATUK, 2007, p.33).

Um exemplo dado por Larry Swatuk sobre a reação desencadeada pelas mudanças climáticas que pode ser considerada, sob o ponto de vista da segurança, como ameaça é o caso da região do rio Chalimbana, na Zâmbia. Esta região possui algumas represas que servem de suporte às atividades econômicas das fazendas maiores da região e que, nos últimos anos, tem sido palco de constantes contestações em face das mudanças climáticas, que fazem com que estas áreas represadas reduzam a capacidade dos pequenos fundiários de exercer atividades agrícolas. Segundo Swatuk, o estudo deste caso serve como modelo de compreensão para vários outros casos da região da SADC (SWATUK, 2007, p. 25-26).

Assim, com o surgimento desses pequenos conflitos na região em razão das modificações econômicas decorrentes das alterações climáticas, podemos verificar que torna-se maior o impacto ambiental (e consequentemente a possibilidade de conflitos) em razão do baixo grau de resiliência da região. A escolha feita neste trabalho para ressaltar este grau de resiliência é por meio do IDH.

O IDH, ou Índice de Desenvolvimento Humano, é um índice utilizado pelo PNUD/ONU para servir de referência de desenvolvimento social e econômico (PNUD, 2013). Este índice é uma medida de desenvolvimento com base em três indicadores combinados: 
expectativa de vida, nível educacional e renda. Este índice varia em uma escala de 0 a 1 , sendo 1 o mais alto grau de desenvolvimento possível. (PNUD, 2013)

Os países são, com base neste índice, classificados em quatro categorias de desenvolvimento: muito elevado, países com IDH superior a 0.796; elevado, países com IDH entre 0.710 e 0.796; médio, para países com índice entre 0.534 e 0.710 ; e baixo, para países com IDH inferior a 0.534. Estes valores foram extraídos do documento de 2013 (PNUD, 2013). Sugere-se, assim que a versão de 2014 for publicada - a data prevista para a publicação é 24 de julho de 2014 que este estudo seja novamente refeito.

A tabela abaixo irá mostrar o IDH dos membros da SADC, de acordo com o resultado de 2013: 
TABELA 2 - IDH dos países da SADC:

\begin{tabular}{ccc}
\hline País & IDH & Ranking (187 países) \\
\hline Angola & 0.508 & 148 \\
Botswana & 0.634 & 119 \\
República Democrática do & 0.304 & 186 \\
Congo & & \\
Lesoto & 0.461 & 158 \\
Madagascar & 0.483 & 151 \\
Malawi & 0.418 & 170 \\
Ilhas Maurício & 0.737 & 80 \\
Moçambique & 0.327 & 185 \\
Namíbia & 0.608 & 128 \\
Seychelles & 0.806 & 46 \\
África do Sul & 0.629 & 121 \\
Suazilândia & 0.536 & 141 \\
Tanzânia & 0.476 & 152 \\
Zâmbia & 0.448 & 163 \\
Zimbabwe & 0.397 & 172 \\
\hline
\end{tabular}

Fonte: PNUD, 2013b, p.16-19.

Observa-se aqui que, com exceção de três países da organização (Botswana, Ilhas Maurício e Seychelles), todos os outros estão classificados como sendo países de baixo desenvolvimento.

A correlação entre IDH e conflitos foi verificada por Katharine Cornish, em um trabalho que afirma que a relação entre pobreza e 
conflito está relacionada à fraqueza do Estado - sendo esta última definida em seu artigo como incapacidade de realizar "responsabilidades fundamentais relacionadas à segurança, serviço, instituições governamentais e economia" (CORNISH, 2013).

Assim, observa-se que, em face às variadas possibilidades de alteração climáticas que causam depreciações econômicas, em conjunto com a baixa resiliência decorrente da fraqueza dos Estados-membro, cujas propensões a conflitos são grandes, pode-se perceber que há fortes indícios de possibilidades de analisar-se a questão ambiental na região como variável relevante para ameaças de segurança.

\section{Considerações finais}

As mudanças no sistema internacional ocorridas no pós-Guerra Fria alteraram a configuração mundial, com inovações tanto nas possibilidades de análise por parte dos teóricos da segurança quanto aos assuntos presentes na pauta da segurança. Este trabalho tenta trazer, com base nessas mudanças, uma discussão frutífera acerca das novas abordagens sobre o conceito de ameaça, que hoje pode englobar muito mais temas do que era possível anteriormente.

Assim, enquanto outrora era leviano tratar de vetores de causalidade como economia e meio-ambiente para questões de segurança (segundo algumas vertentes teóricas predominantes), hoje tal 
fato não pode ser desprezado. Ante a esse contexto, observando-se tanto a mudança paradigmática relativa ao conceito de ameaça, bem como às reformulações teóricas propostas em relação ao papel do Estado e do posicionamento da sociedade civil em relação a este (desdobramentos do hiperconflito de Mittelman (2010)), discussões teóricas mais profundas sobre a relação Estado-indivíduo se fazem necessárias.

Deste modo, este trabalho, ao abordar o binômio meioambiente - segurança, apresenta uma possibilidade teórica de análise da relação Estado-indivíduo. Conforme descrito acima, este trabalho analisa, por meio de documentos que tratam de variações climáticas recentes na região das SARC, possibilidades de que estas variações transformem-se em conflitos. Tal análise pode ser mais bem aprofundada, na medida em que se obtenham ainda mais informações sobre as tendências mais atuais referentes à situação climática da região.

$\mathrm{O}$ uso do IDH serviu para sustentar que, embora as variações climáticas existam e exerçam efeitos em vários locais do mundo, as reduzidas capacidades dos Estados da região da SARC, traduzidos em uma menor resiliência, aumentam a probabilidade de que consequências sócio-econômicas das variações climáticas possam escalar para conflitos intra ou interestatais. 


\section{Referências bibliográficas:}

BARROS-PLATIAU, Ana Flávia; VARELLA, Marcelo Dias; SCHLEICHER, Rafael T. Meio ambiente e relações internacionais: perspectivas teóricas, respostas institucionais e novas dimensões de debate. In: Rev. bras. polít. int. [online]. 2004, vol.47, n.2, pp. 100130. Disponível em: http://www.scielo.br/pdf/rbpi/v47n2/v47n2a04.pdf

BAUMAN, Zygmunt. Medo líquido. Rio de Janeiro: Zahar, 2008.

BISWAS, Niloy R. Is environment a security threat? Environmental security beyond securitization In: International affairs review, vol. XX no. 1, winter 2011.

CORNISH, Katherine. Poverty and armed conflict: why state capacity matters most. 2013. Disponível em http://www.eir.info/2013/02/06/poverty-and-armed-conflict-why-state-capacitymatters-most/.

FALK, Richard. The declining world order: America's imperial geopolitics. New York: Routledge, 2004.

FOUCAULT, Michel. Em defesa da sociedade: curso no Collège de France (1975-1976). São Paulo: WMF Martins Fontes, 2010. 
. Nascimento da biopolítica: curso dado no Collège de France (1978-1979). São Paulo: Martins Fontes, 2008.

MATTHEW, Richard; MCDONALD, Bryan. Networks of threats and vulnerability: lessons from environmental security research. In: Environmental Change and Security Program, 2004. Report... Washington: Wilson Center, 2004, p.36-42.

MEDEIROS, M.; STEINER, A. Como saber se as Convenções Internacionais de meio-ambiente realmente funcionam? Abordagens teórico-metodológicas sobre a eficácia dos regimes ambientais. In: Contexto Internacional, vol. 32, n. 2, julho/dezembro 2010.

MITTELMAN, James. Hyperconflict: globalization and insecurity. Stanford: Stanford University Press, 2010.

PNUD. Human development index (HDI). 2013. Disponível em http://hdr.undp.org/en/statistics/hdi. Acesso em 29 jun. 2014.

. Human Development Report 2013 - The rise of the South: human progress in a diverse world. 2013. Disponível em: http://hdr.undp.org/sites/default/files/hdr2013_en_summary.pdf. Acesso em 29 jun. 2014.

SADC. Treaty of the Southern African Development Community. Namíbia, 1992. 
Member states. 2012. Disponível em:

http://www.sadc.int/member-states. Acesso em 29 jun. 2014.

SARTRE, Jean Paul. Critique de la raison dialectique. Poitiers:

Editions Gallimard, 1960.

SWATUK, Larry A. Regionalexpertise - Southern Africa,

Environmental Change and Regional Security: An Assessment. In:

Wissenschaftlicher Beirat der Bundesregierung Globale

Umweltveränderungen, 2007. Hauptgutachten... Berlin: SpringerVerlag, 2007.

VILLA, Rafael Duarte. Atores não-estatais e meio ambiente nas relações internacionais: Greenpeace e a Antártica. In: Desenvolvimento e Meio Ambiente, n. 4, p. 45-57, jul./dez. 2001. Curitiba: Editora da UFPR, 2001. 